\title{
Comprehensive analysis of gene expression profiles provides insight into the pathogenesis of Crohn's disease
}

\author{
LUMIN BO ${ }^{1}$, HONGYU FU ${ }^{1}$ and JUNCHI YANG ${ }^{2}$ \\ Departments of ${ }^{1}$ Gastroenterology and ${ }^{2}$ Gastrointestinal Surgery, Changhai Hospital, Shanghai 200433, P.R. China
}

Received December 3, 2017; Accepted May 18, 2018

DOI: $10.3892 / \mathrm{mmr} .2018 .9267$

\begin{abstract}
Crohn's disease (CD) is a type of inflammatory bowel disease that cannot be fully cured by medication or surgery. In the present study, the aim was to understand the underlying mechanisms of CD. Two CD microarray datasets were downloaded from The Gene Expression Omnibus database: GSE36807 (13 CD and 7 normal samples) and GSE59071 (8 CD and 11 normal samples). A series of bioinformatics analyses were conducted, including weighted gene co-expression network analysis to identify stable modules, and analysis of differentially expressed genes (DEGs) between CD and normal samples. The common DEGs in the GSE36807 and GSE59071 datasets were screened. Subsequently, overlapping genes in the stable modules and the DEGs were selected to construct a protein-protein interaction (PPI) network using Cytoscape software. Enrichment analysis of genes in the network was performed to explore their biological functions. A total of 10 stable modules and 927 DEGs were identified, of which 234 genes were shared in the stable modules and the DEGs. After removal of 32 uncharacterized genes, 202 genes were selected to build the PPI network. Low density lipoprotein receptor (LDLR), toll-like receptor 2 (TLR2), lipoprotein lipase (LPL), forkhead box protein M1 (FOXM1) and neuropeptide Y (NPY) were revealed as key nodes with high degree. Pathway enrichment analysis demonstrated that $L P L$ was enriched in the peroxisome proliferator-activated receptor (PPAR) signaling pathway. In conclusion, LDLR, TLR2, FOXM1 and $N P Y$, as well as $L P L$ in the PPAR signaling pathway may serve critical roles in the pathogenesis of CD.
\end{abstract}

\section{Introduction}

Crohn's disease (CD) is a type of inflammatory bowel disease (IBD) that can affect any part of the gastrointestinal tract (1). This disease is caused by immune, bacterial and environmental

Correspondence to: Dr Junchi Yang, Department of Gastrointestinal Surgery, Changhai Hospital, 168 Changhai Road, Shanghai 200433, P.R. China

E-mail: yjc990@outlook.com

Key words: Crohn's disease, weighted gene co-expression network analysis, meta-analysis, protein-protein interaction network, enrichment analysis factors in individuals with genetic susceptibility (2-4). Patients with CD usually suffer from weight loss, abdominal pain, fever and diarrhea (5); other complications associated with CD include arthritis, anemia, inflammation of the eye, fatigue and skin problems (6). CD is a common disease in the developed world, with increasing incidence and prevalence in developing countries $(7,8)$. At present, there are no medical treatments or operative interventions that can fully cure $\mathrm{CD}$, and in some cases surgery may even result in recurrence of the disease (9). Therefore, the underlying mechanisms of CD need to be explored in order to develop novel treatment strategies.

So far, some progress has been made with understanding the pathogenesis of CD. For example, it was demonstrated that mutation of the autophagy-related 16-like 1 gene is associated with $\mathrm{CD}$, and may possibly support the role of autophagy in the development and progression of IBD (10). T helper (Th) 17 cells are a unique $\mathrm{Th}$ cell lineage that serve an important role in inflammatory diseases by secreting proinflammatory cytokines, including interleukin 17 (IL-17) and IL-23, and may mediate the Th1/Th17 imbalance seen in CD and ulcerative colitis $(11,12)$. A previous study reported that signal transducer and activator of transcription 3 (STAT3) and Janus kinase 2 (JAK2), which are involved in the STAT-JAK pathway, can increase the risk of CD (13). Nucleotide-binding oligomerization domain-containing 2 (NOD2) is expressed throughout the small intestine, with highest expression in the terminal ileum where there is an abundance of Paneth cells, and is also closely associated with pathogenesis of CD $(14,15)$. Despite these profound findings, pathogenesis of CD has not been fully elucidated.

Bioinformatics analysis is a useful tool for exploring disease pathogenesis and screening novel therapeutic targets (16). Kenny et al performed a genome-wide association study in an Ashkenazi Jewish population with CD and revealed that 16 replicated and novel loci made up $11.2 \%$ of the total genetic variations associated with CD risk (17). Fransen et al employed expression quantitative trait loci to select single nucleotide polymorphisms (SNPs) for follow-up and identified two CD-associated SNPs in the ubiquitin-conjugating enzyme E2L 3 and B-cell chronic lymphocytic leukemia/lymphoma 3 genes (18). These findings provide some information on the etiology of CD; however, to the best of our knowledge the mechanisms of CD pathogenesis have not yet been investigated by comprehensive bioinformatics analysis.

In the present study, comprehensive bioinformatics analysis was carried out to understand the pathogenesis of CD and 
identify novel therapeutic targets. The gene expression profiles of patients with $\mathrm{CD}$ were downloaded from a public database, and subsequently a series of bioinformatics analyses were performed, including weighted gene co-expression network analysis (WGCNA), meta-analysis, protein-protein interaction (PPI) network analysis and enrichment analysis for key genes associated with $\mathrm{CD}$. This study contributes towards the further understanding of the mechanisms underlying human $\mathrm{CD}$.

\section{Materials and methods}

Data source. The key words 'Crohn's disease' and 'Homo sapiens' were used to search relevant gene expression profile data on the Gene Expression Omnibus (GEO) database (http://www.ncbi.nlm.nih.gov/geo/). The inclusion criteria were as follows: i) Gene expression profiles; ii) intestinal tissues from patients with $\mathrm{CD}$ (not cells); iii) availability of both $\mathrm{CD}$ and normal samples; iv) human samples and v) number of total samples $\geq 18$. Based on these criteria, two microarray datasets were selected: GSE36807 (Platform GPL570; 13 CD and 7 normal samples) (19) and GSE59071 (Platform GPL6244; $8 \mathrm{CD}$ and 11 normal samples) (20).

Data pre-processing. The raw data (CEL files) were downloaded from GEO. Subsequently, the oligo package version 1.41 .1 from $R$ (http://www.bioconductor.org/packages/release/bioc/html/oligo. html) (21) was utilized to perform pre-treatments, including conversion of data format, summarization using median-polish, background correction using the MAS method and data normalization by quantile method.

WGCNA analysis. WGCNA is a popular systems biology tool used to construct gene co-expression networks, which can be used to detect disease-associated gene clusters and identify therapeutic targets (22). GSE36807 and GSE59071 were used as the training and validation sets, respectively. Stable gene modules associated with $\mathrm{CD}$ were screened using the R WGCNA package version 1.61 (https://cran.r-project. org/web/packages/WGCNA/index.html) (22), with the following parameters; cutHeight $=0.95$ and $\geq 30$ genes/module; the correlation coefficients (CC) were also calculated.

Meta-analysis. Using the MetaDE.ES function in the $\mathrm{R}$ MetaDE package (https://cran.r-project. org/src/contrib/Archive/MetaDE/) (23), differentially expressed genes (DEGs) between CD and normal samples that were common to the two datasets were identified. To ensure homogeneity of genes, $\operatorname{tau}^{2}=0$, Qpval $>0.05$ and false discovery rate $<0.05$ were used as the cut-off criteria.

Construction of the PPI network. Genes in the stable modules and DEGs were compared, and overlapping genes were used as candidate genes for construction of the PPI network. An integrated PPI network was generated from the candidate genes by merging numerous PPI databases, including BioGRID version 3.4.153 (http://thebiogrid.org/) (24), Human Protein Reference Database release 9 (http://www.hprd.org/) (25) and STRING version 10.5 (https://string-db.org/) (26). The PPI network was visualized using the Cytoscape software version 3.3.0 (http://www.cytoscape.org/) (27).
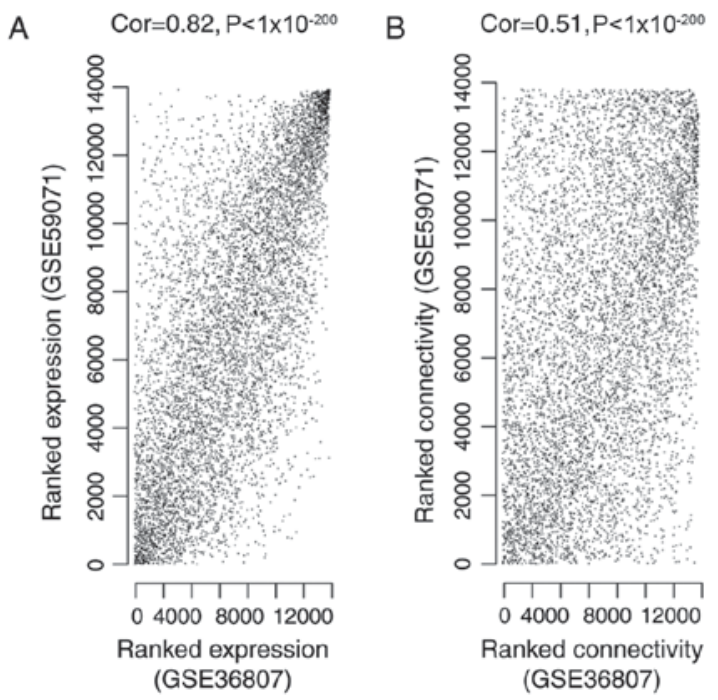

Figure 1. Correlation between (A) gene expression and (B) gene connectivity in the GSE36807 and GSE59071 datasets. Cor, correlation.

Functional and pathway enrichment analyses. The Database for Annotation, Visualization and Integrated Discovery (DAVID) version 6.8 (https://david.ncifcrf.gov/) is an easy-to-use web tool in which genes are annotated and summarized according to shared categorical data (28). Based on DAVID analysis, Gene Ontology (GO) (29) functional enrichment analysis and Kyoto Encyclopedia of Genes and Genomes (KEGG) (30) pathway enrichment analysis were conducted for the nodes (a node represents a gene) identified in the PPI network, with the criterion of $\mathrm{P}<0.05$.

\section{Results}

WGCNA analysis. To ensure the GSE36807 and GSE59071 datasets were comparable, correlation of gene expression and connectivity of common genes was analyzed. The results revealed that the $\mathrm{CC}$ for gene expression and connectivity between the two datasets were $0.82\left(\mathrm{P}<1 \times 10^{-200} ;\right.$ Fig. $\left.1 \mathrm{~A}\right)$ and $0.51\left(\mathrm{P}<1 \times 10^{-200}\right.$; Fig. 1B), respectively. Therefore, it was deduced that the GSE36807 and GSE59071 datasets were comparable.

To meet the prerequisite of scale-free network distribution, the value of the adjacency matrix weighting parameter 'power' was explored. After setting the ranges of parameters for the network, the scale-free topology matrix was calculated. Using GSE36807 as the essential data, the scale-free topology model fit was calculated and statistics were selected for graphing (Fig. 2A). The greater the value of $\mathrm{CC}^{2}$, the closer the network was to scale-free distribution. Based on this theory, the mean connectivity of genes was calculated using power $=8$ as this was the minimum power value to achieve $R^{2}=0.9$. The results demonstrated that the mean connectivity was 16 , which conforms to the node connection properties of a scale-free network (Fig. 2B).

Using GSE36807 as the training set, disease-associated modules were screened. Variation in gene expression in the samples was calculated, and the genes with variation coefficients $>0.1$ were selected. Subsequently, the community dissimilarities among the genes were calculated, and a clustering tree was obtained. A total of 10 modules (D1M1, D1M2, D1M3, D1M4, D1M5, D1M6, D1M7, D1M8, D1M9 and D1M10) 
Table I. Correlation of gene expression for the same color modules in GSE36807 and GSE59071.

\begin{tabular}{|c|c|c|c|c|c|c|c|}
\hline \multicolumn{2}{|c|}{ Modules } & \multirow[b]{2}{*}{ Color } & \multirow[b]{2}{*}{ Size } & \multicolumn{3}{|c|}{ Module preservation } & \multirow[b]{2}{*}{ Module characterization } \\
\hline GSE36807 & GSE59071 & & & Correlation & P-value & Z-score & \\
\hline D1M1 & D2M1 & Black & 163 & 0.40 & $1.2 \times 10^{-7}$ & 20.34 & Cell adhesion \\
\hline D1M2 & $\mathrm{D} 2 \mathrm{M} 2$ & Blue & 431 & 0.37 & $2.0 \times 10^{-15}$ & 10.05 & Cell adhesion \\
\hline D1M3 & $\mathrm{D} 2 \mathrm{M} 3$ & Brown & 412 & 0.30 & $5.1 \times 10^{-10}$ & 15.88 & Cell-cell signaling \\
\hline D1M4 & $\mathrm{D} 2 \mathrm{M} 4$ & Green & 180 & 0.50 & $8.9 \times 10^{-13}$ & 16.73 & Immune response \\
\hline D1M5 & D2M5 & Grey & 368 & 0.35 & $4.8 \times 10^{-12}$ & 4.12 & - \\
\hline D1M6 & D2M6 & Magenta & 38 & 0.32 & $5.0 \times 10^{-2}$ & 11.74 & Cell cycle \\
\hline D1M7 & D2M7 & Pink & 123 & 0.41 & $2.5 \times 10^{-6}$ & 5.53 & Regulation of transcription \\
\hline D1M8 & $\mathrm{D} 2 \mathrm{M} 8$ & Red & 177 & 0.35 & $1.8 \times 10^{-6}$ & 21.44 & Cell adhesion \\
\hline D1M9 & D2M9 & Turquoise & 944 & 0.68 & $4.2 \times 10^{-129}$ & 17.93 & Ion transport \\
\hline D1M10 & D2M10 & Yellow & 352 & 0.42 & $1.8 \times 10^{-16}$ & 29.51 & Immune response \\
\hline
\end{tabular}

Size is the number of genes in one module. Module characterization is the significant functional term for each module. $5<Z<10$ and $\mathrm{Z}>10$ indicate stable and highly stable, respectively. D1, Dataset1 GSE36807; D2, Dataset2 GSE59071; M, module.

A

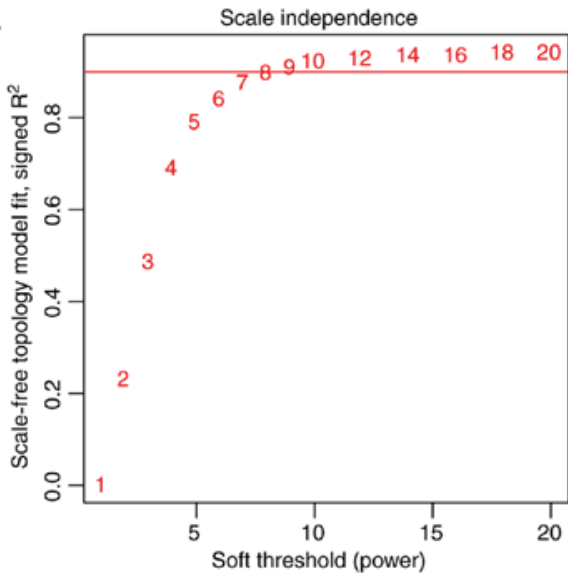

B

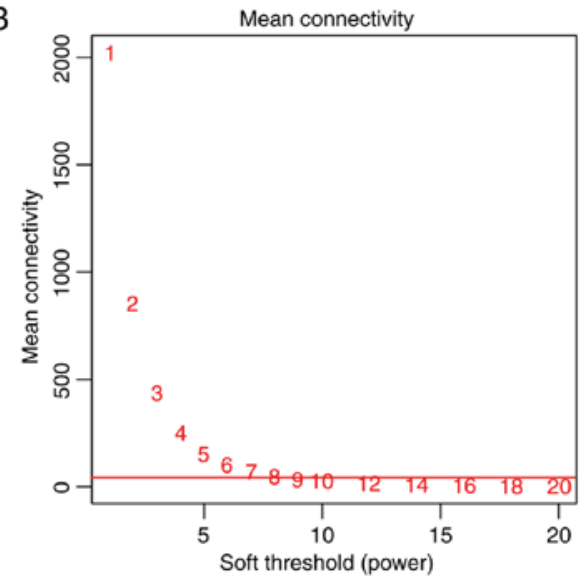

Figure 2. (A) Selection of the weighting parameter 'power'. The red line indicates when the square of correlation coefficient equals 0.9. (B) Mean connectivity of genes according to 'power'. The red line indicates a mean connectivity of 16 when the 'power' is 8 .

were identified using cutHeight=0.95 (Fig. 3A). Similarly, module division was performed for GSE59071 (Fig. 3B) and the modules in this dataset were used to evaluate the stability of those identified in the training set.

The correlation of gene expression was analyzed for the same color modules in the two datasets, and the results demonstrated that 10 modules were stable (Table I; preservation $\mathrm{Z}$-score $>5 ; \mathrm{P} \leq 0.05$ ). According to the expression similarities of the module genes, the correlation of the modules in GSE36807 (Fig. 4A) and GSE59071 (Fig. 4B) were analyzed (Table I). By combining the correlations among the modules with the GO categories for genes in the stable modules (Table I), a network for the modules was constructed (Fig. 4C). This revealed that genes in three stable modules (M1, M2 and M8) were significantly associated with cell adhesion and genes in two stable modules (M4 and M10) were significantly associated with immune response.

Meta-analysis. After calculation of the parameter values of each gene, a total of 927 DEGs were screened according to

\section{A Gene dendrogram and module colors (GSE36807)}

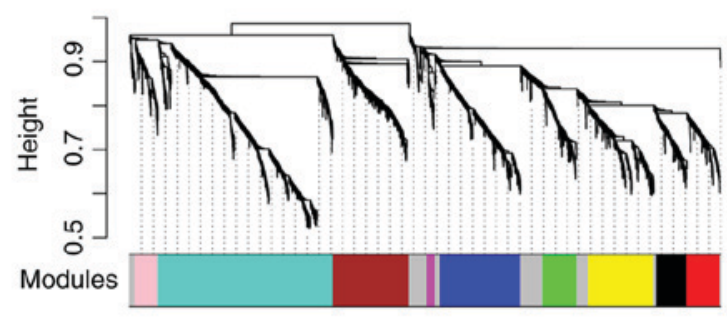

B Gene dendrogram and module colors (GSE59071)

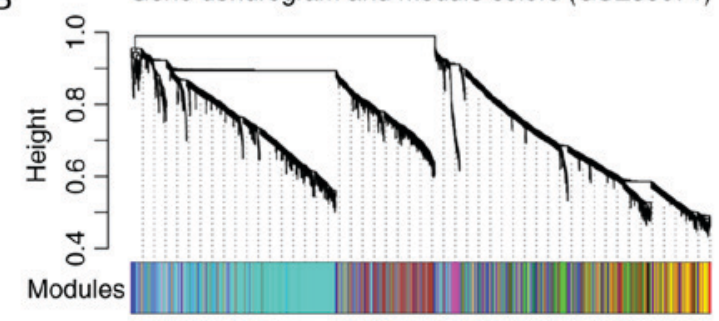

Figure 3. Module partition trees of (A) GSE36807 and (B) GSE59071; each color represents a different module. 
A

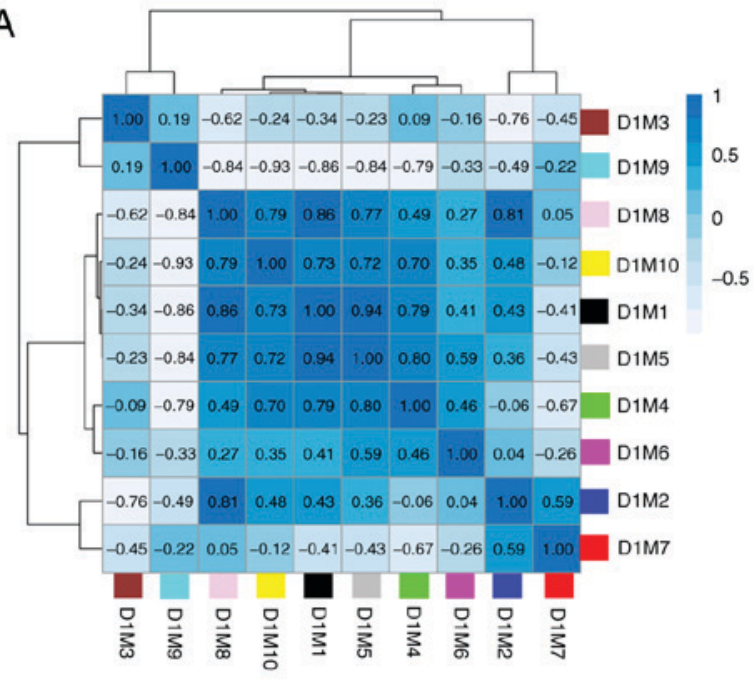

B

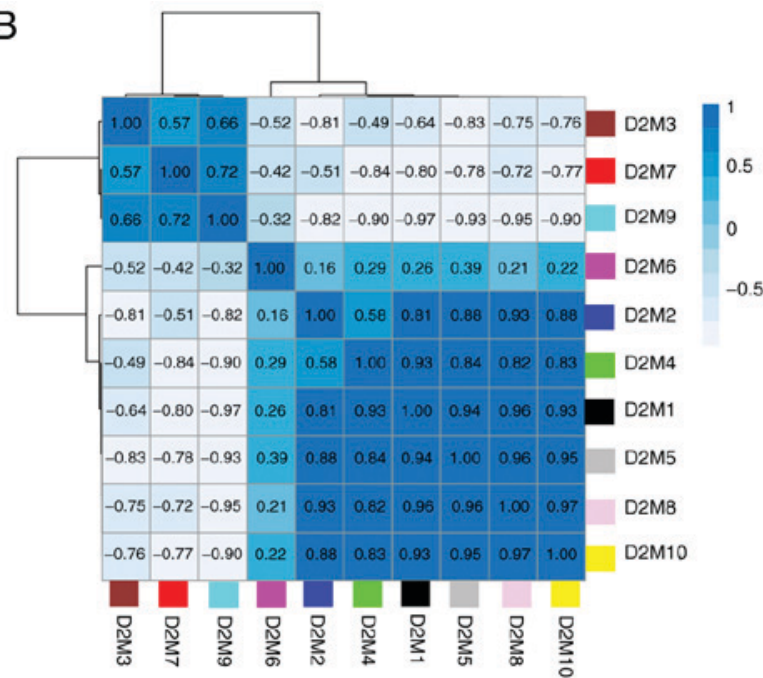

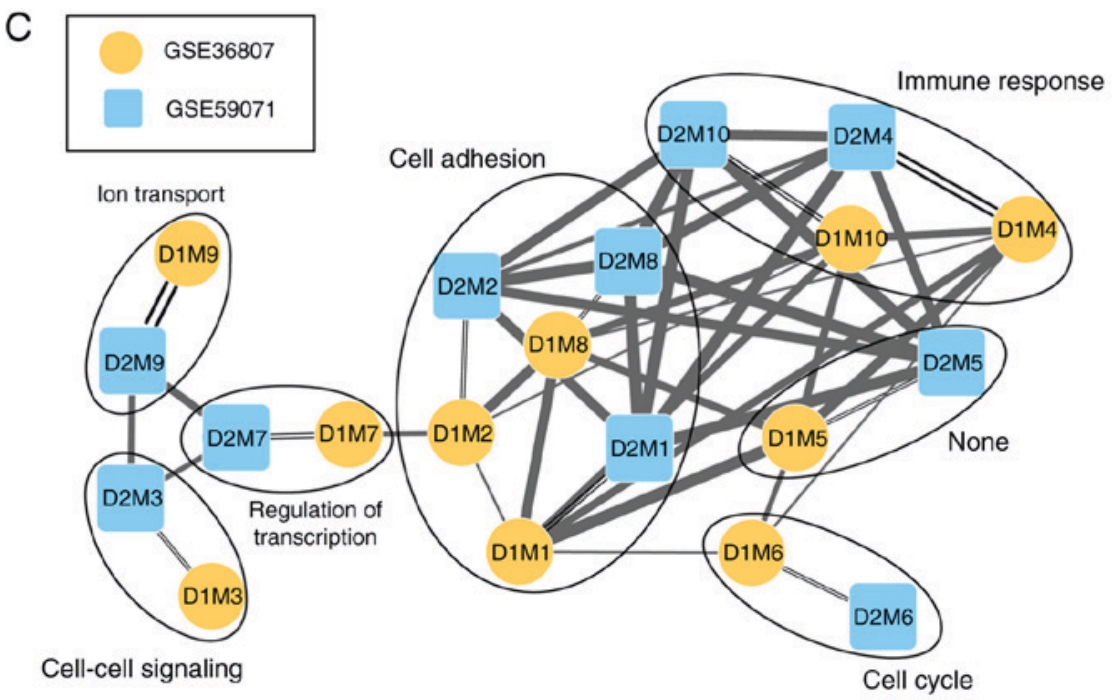

Figure 4. Correlation cluster diagrams for the modules of (A) GSE36807 and (B) GSE59071. (C) Module-module correlation network. Single lines connect modules within the same dataset and double lines connect modules across datasets. The thickness of the links indicates the extent of correlation. D1, dataset 1 GSE36807; D2 dataset 2 GSE59071; M, module.

the aforementioned thresholds. The heatmap revealed that changes in expression of the DEGs were similar for GSE36807 and GSE59071 (Fig. 5).

Construction of the PPI network. A total of 234 overlapping genes from genes in the stable modules (3,188 genes) and the 927 DEGs were identified and used as candidate genes (Fig. 6A). The number and proportion of these genes in different modules are shown in Fig. 6B. A total of 32 uncharacterized genes in the grey module were removed from subsequent analyses. PPIs were predicted for the remaining 202 genes and a PPI network (123 nodes and 270 edges) was constructed (Fig. 7). Notably, low density lipoprotein receptor (LDLR; node degree $=22$ ), toll-like receptor 2 (TLR2; node degree $=19$ ), lipoprotein lipase (LPL; node degree=18), forkhead box M1 (FOXM1; node degree=17) and neuropeptide Y (NPY; node degree=16) were the top five nodes with high degrees in the PPI network.

Functional and pathway enrichment analyses. A total of $24 \mathrm{GO}$ categories and $12 \mathrm{KEGG}$ pathways were enriched for the PPI network nodes. Notably, the network nodes were mainly

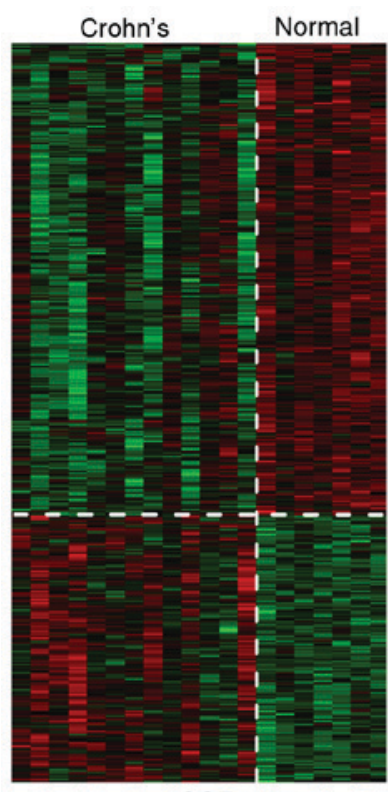

GSE36807

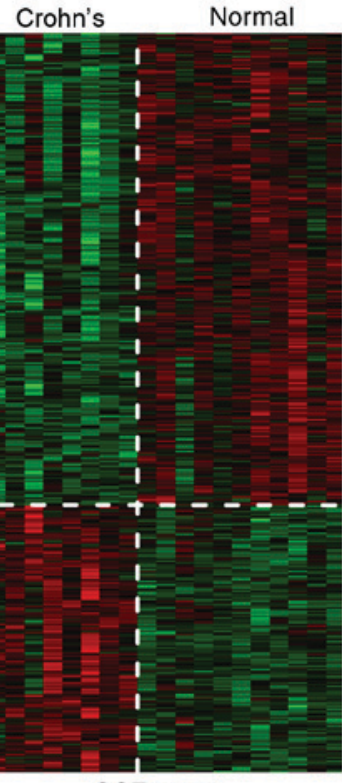

GSE59071
Figure 5. Clustering heatmap of differentially expressed genes. 
A

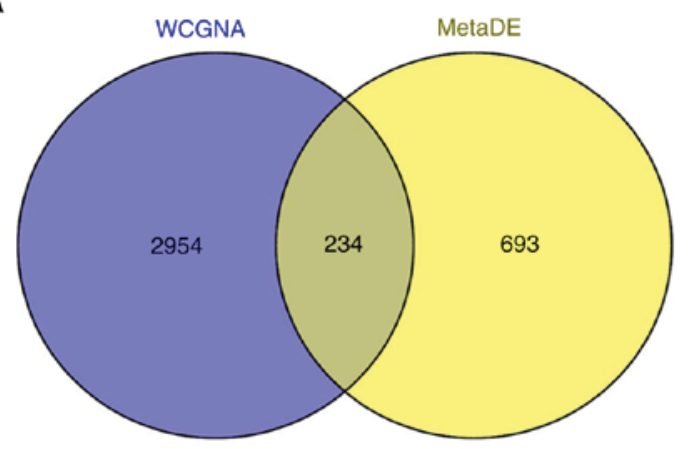

B

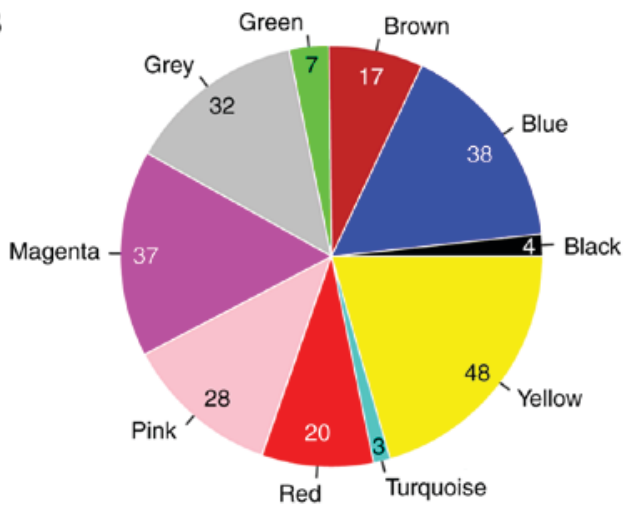

Figure 6. (A) Venn diagram depicting the overlap between the 3,188 genes in the stable modules and the 927 differentially expressed genes. (B) Pie chart of the number of genes in different modules. WCGNA, weighted gene co-expression network analysis.

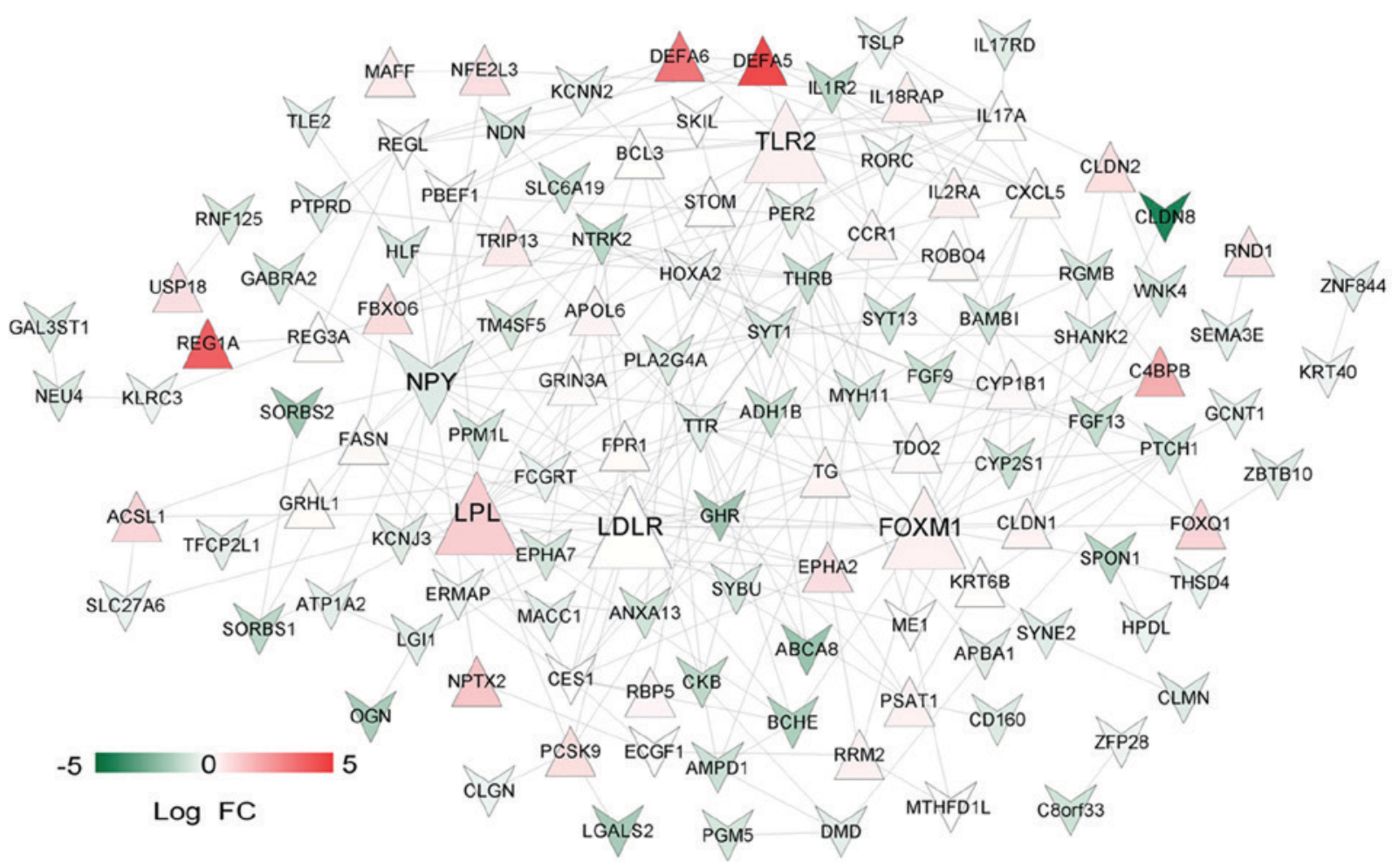

Figure 7. Protein-protein interaction network. Upright red and inverted green triangles represent upregulated and downregulated proteins, respectively. The color intensity indicates gene expression. The enlarged nodes are the top five nodes with highest degrees. FC, fold change.

implicated in the enzyme-linked receptor protein signaling pathway ( $\mathrm{GO}$ category; $\mathrm{P}=0.00185)$, cell-cell signaling $(\mathrm{GO}$ category; $\mathrm{P}=0.003505)$, peroxisome proliferator-activated receptor (PPAR) signaling pathway (KEGG pathway; $\mathrm{P}=0.001014)$ and cytokine-cytokine receptor interaction (KEGG pathway; $\mathrm{P}=0.004187$ ) (Fig. 8).

\section{Discussion}

In the present study, a total of 10 stable CD-associated modules were identified using WGCCA; of which three stable modules (M1, M2 and M8) and two stable modules (M4 and M10) were significantly associated with cell adhesion and immune response, respectively. In addition, 927 overlapping DEGs in the GSE36807 and GSE59071 datasets were screened. In the
PPI network, five important nodes with relatively high node degrees were identified as LDLR, TLR2, LPL, FOXM1 and NPY.

Previous studies have investigated the relationship between inflammatory cytokines and $\operatorname{LDLR}(31,32)$, and demonstrated that the inflammatory cytokines IL- $1 \beta$ and tumor necrosis factor (TNF)- $\alpha$ can regulate $L D L R$ expression leading to accumulation of high concentrations of low-density lipoprotein (33). FOXM1 belongs to the forkhead box (FOX) family of transcription factors, and regulates key genes involved in goblet cell metaplasia and lung inflammation $(34,35)$. FOXM1 is an important mediator of cell proliferation, and its expression is enhanced during gastric carcinogenesis induced by Helicobacter pylori infection (36). Overexpression of triglyceride-rich lipoproteins can induce lipid accumulation and early inflammatory responses, 
A
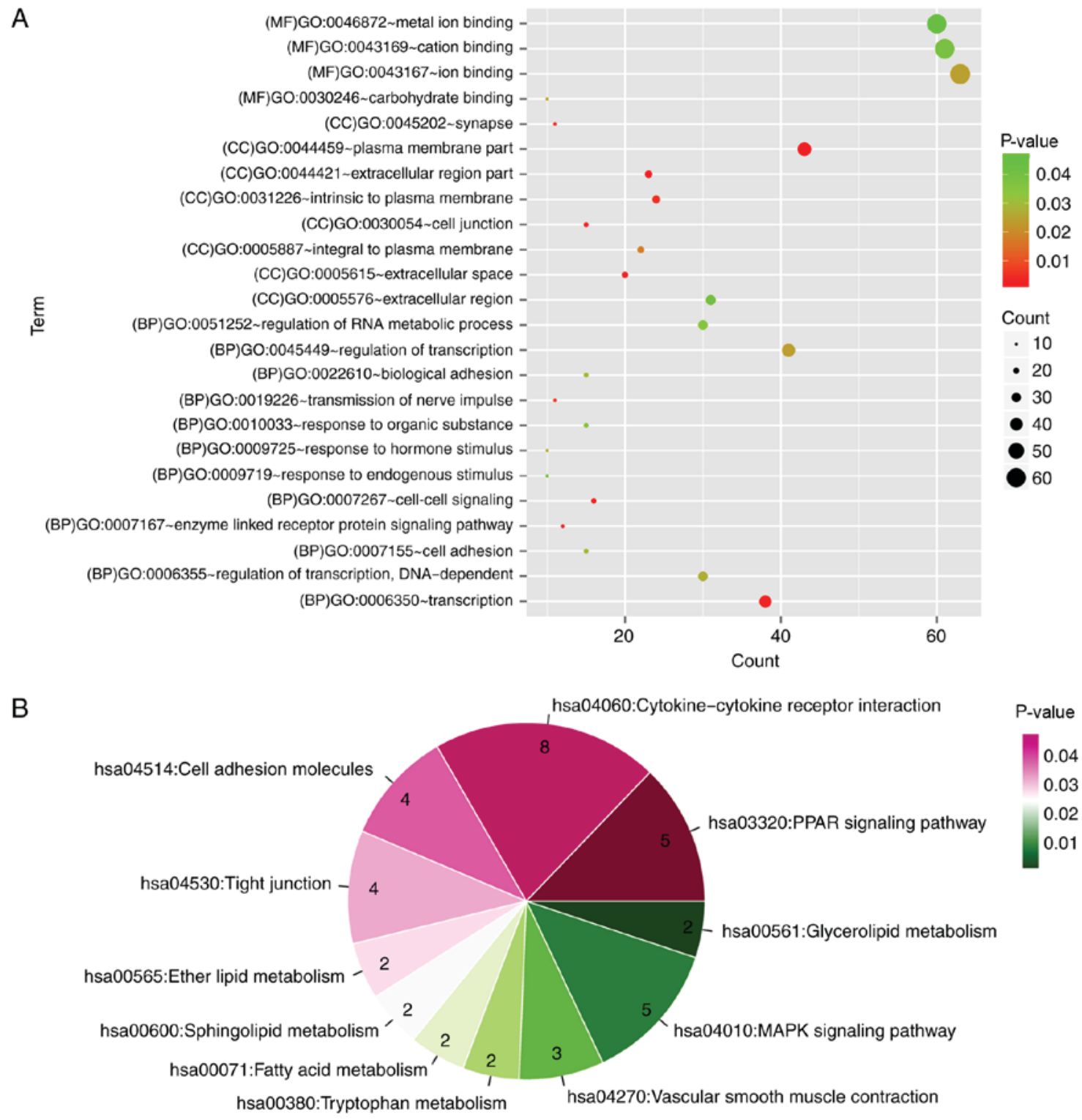

Figure 8. (A) Functional categories and (B) pathways enriched for the protein-protein interaction network nodes. BP, biological process; CC, cellular component; GO, Gene Ontology; MAPK, mitogen-activated protein kinase; MF, molecular function; PPAR, peroxisome proliferator-activated receptor.

and LPL serves an anti-inflammatory role by hydrolyzing triglyceride-rich lipoproteins (37). PPARs can regulate lipid metabolism-associated genes and control inflammation, and PPAR $\alpha$ and $-\gamma$ activators contribute to macrophage secretion of LPL $(38,39)$. At present, there is no direct evidence for the involvement of LDLR, FOXM1 and LPL in CD development. However, their expression levels may be linked to disease progression. A study demonstrated that polymorphisms of LIGHT (a TNF superfamily member) are associated with $\mathrm{CD}$, and inhibiting $L I G H T$ reduces dyslypidemia in mice lacking LDLR (40). This suggests a possible association between $L D L R$ and CD. In IBD, the susceptibility genes include FYN proto-oncogene and HCK proto-oncogene, which are involved in the phosphoinositide 3-kinase signaling network, which also contains FOXMI (41). Since CD is a major subtype of IBD, FOXM1 may also be associated with $\mathrm{CD}$. With regards to the $L P L$ gene, decreased expression is observed in $\mathrm{CD}$ adipose-derived stem cells (ASCs) independent of clinical stage, compared with in healthy ASCs, indicating that CD modifies the plasticity of mesenteric ASCs (42). In the present study, LPL was upregulated in CD PPI network, indicating a potential role of $L P L$ in the progression of $\mathrm{CD}$; pathway enrichment analysis revealed that $L P L$ was enriched in the PPAR signaling pathway. Interestingly, the alterations in LPL expression levels of ASC cells and CD tissues revealed different trends, suggesting that the creeping fat tissue may be associated with the immunomodulatory properties in patients with $\mathrm{CD}$. Overall, the results demonstrated that increases in LDLR, FOXMI and $L P L$ may be highly relevant to the development of $\mathrm{CD}$.

TLRs initiate immune responses to microbial infection, with TLR2 recognizing bacterial lipoproteins, zymosan, peptidoglycan and lipoteichoic acids $(43,44)$. TLR2, TLR4, and their transmembrane co-receptor cluster of differentiation 14 , are all upregulated in the terminal ileum of patients with IBD, and their dysregulation may serve critical roles in the pathogenesis of IBD (45). NOD2 mediates the anti-inflammatory cytokine bias induced by TLR2 ligands; therefore, defective NOD2 function may promote inflammation and increase the disease 
risk of CD (46). TLR2 and TLR4 have been reported to be overexpressed in the inflamed colonic mucosa of children with IBD, indicating that innate immunity is associated with the pathogenesis of IBD (47). A previous study discovered that TLR2 and TLR4 are highly expressed on inflammation-associated intestinal macrophages (IMACs), leading to a higher reactivity to lipopolysaccharide and possibly CD (48). In addition, the role of Gp96, an endoplasmic reticulum chaperone for multiple protein substrates, in $\mathrm{CD}$ has been investigated. The results demonstrated that the lack of Gp96 in CD IMACs is associated with loss of tolerance against the host gut flora, and that the Gp96 knockdown cell line has decreased TLR2 and TLR4 expression (49). Therefore, it may be hypothesized that TLR2 has an important role in the pathogenesis of CD. Neurogenic inflammation is critical for the development of IBD, and NPY induces oxidative stress and colitis by regulating the expression of neuronal nitric oxide synthase (50). NPY and the NPY receptor Y1 (NPY1R) are associated with intestinal inflammation and suppression of NPY1R signaling may represent a potential therapeutic strategy for colonic inflammation $(51,52)$. NPY peptides have been implicated in various gastrointestinal disorders, including IBD, malabsorption and short gut; and NPY receptor agonists and antagonists can be used for preventing diarrhea and intestinal inflammation (53). Gut neurohormones, including NPY, can affect inflammation by interacting with the immune system, and NPY represents a promising target for treating IBD (54). In the present study, NPY was reported as an important node with a high degree in the CD PPI network; however, the expression levels of NPY were lower in the CD samples of patients compared with in the healthy control, which is inconsistent to the reports $(50,52)$. Considering the important role of NPY in CD and that its expression levels varies from that of previous reports, further validation of NPY mRNA and protein expression in clinical samples is required.

Nevertheless, there are some limitations to the present study. Firstly, the results are solely based on bioinformatics analysis and although various important genes for CD etiology were identified, experimental validation is required. Secondly, only two CD microarray datasets were selected with relatively small sample sizes; therefore, this could affect the robustness of the results. Further experiments will be designed and conducted to validate these findings in follow-up studies.

In conclusion, a total of 10 stable modules and 927 DEGs associated with CD were identified. Notably, $L D L R, T L R 2$, FOXM1, NPY and LPL may be the key genes involved in pathogenesis of the disease. $L P L$ may exert its effects through the PPAR signaling pathway.

\section{Acknowledgements}

Not applicable.

\section{Funding}

No funding was received.

\section{Availability of data and materials}

The datasets analyzed during the current study are available from the corresponding author on reasonable request.

\section{Authors' contributions}

LB performed data analyses and wrote the manuscript. HF made substantial contributions to data analyses. JY conceived and designed the present study. All authors read and approved the final manuscript.

\section{Ethics approval and consent to participate}

Not applicable.

\section{Patient consent for publication}

Not applicable.

\section{Competing interests}

The authors declare that they have no competing interests.

\section{References}

1. Baumgart DC and Sandborn WJ: Crohn's disease. Lancet 380: 1590-1605, 2012.

2. Cho JH and Brant SR: Recent insights into the genetics of inflammatory bowel disease. Gastroenterology 140: 1704-1712, 2011.

3. Dessein R, Chamaillard M and Danese S: Innate immunity in Crohn's disease: The reverse side of the medal. J Clin Gastroenterol 42 (Suppl 3): S144-S147, 2008.

4. Stefanelli T, Malesci A, Repici A, Vetrano S and Danese S: New insights into inflammatory bowel disease pathophysiology: Paving the way for novel therapeutic targets. Current Drug Targets 9: 413-418, 2008.

5. Hashash JG, Proksell S and Regueiro MD: Crohn's disease with worsening symptoms. Gastroenterology 145: e5-e6, 2013.

6. Mazor Y, Maza I, Kaufman E, Ben-Horin S, Karban A, Chowers Y and Eliakim R: Prediction of disease complication occurrence in Crohn's disease using phenotype and genotype parameters at diagnosis. J Crohns Colitis 5: 592-597, 2011.

7. Hovde $\varnothing$ and Moum BR: Epidemiology and clinical course of Crohn's disease: Results from observational studies. World J Gastroenterol 18: 1723-1731, 2012.

8. Burisch $\mathbf{J}$ and Munkholm P: Inflammatory bowel disease epidemiology. Curr Opin Gastroenterol 29: 357-362, 2013.

9. Hut'an M and Hut'an Mm: The role of surgery in Crohn's disease treatment. Rozhl Chir 88: 185-188, 2009 (In Slovak).

10. Prescott NJ, Fisher SA, Franke A, Hampe J, Onnie CM, Soars D, Bagnall R, Mirza MM, Sanderson J, Forbes A, et al: A nonsynonymous SNP in ATG16L1 predisposes to ileal Crohn's disease and is independent of CARD15 and IBD5. Gastroenterology 132: 1665-1671, 2007.

11. Kobayashi T, Okamoto S, Hisamatsu T, Kamada N, Chinen H, Saito R, Kitazume MT, Nakazawa A, Sugita A, Koganei K, et al: IL23 differentially regulates the Th1/Th17 balance in ulcerative colitis and Crohn's disease. Gut 57: 1682-1689, 2008.

12. Siakavellas SI and Bamias G: Role of the IL-23/IL-17 axis in Crohn's disease. Discov Med 14: 253-262, 2012.

13. Ferguson LR, Han DY, Fraser AG, Huebner C, Lam WJ, Morgan AR, Duan H and Karunasinghe N: Genetic factors in chronic inflammation: Single nucleotide polymorphisms in the STAT-JAK pathway, susceptibility to DNA damage and Crohn's disease in a New Zealand population. Mutat Res 690: 108-115, 2010.

14. Nabatov AA: The vesicle-associated function of NOD2 as a link between Crohn's disease and mycobacterial infection. Gut Pathog 7: 1, 2015.

15. Balasubramanian I and Gao N: From sensing to shaping microbiota: Insights into the role of NOD2 in intestinal homeostasis and progression of Crohn's Disease. Am J Physiol Gastrointest Liver Physiol 313: G7-G13, 2017.

16. Zhang Y, Szustakowski J and Schinke M: Bioinformatics analysis of microarray data. Methods Mol Biol 573: 259-284, 2009. 
17. Kenny EE, Pe'er I, Karban A, Ozelius L, Mitchell AA, Ng SM, Erazo M, Ostrer H, Abraham C, Abreu MT, et al: A genome-wide scan of Ashkenazi jewish Crohn's disease suggests novel susceptibility loci. PLoS Genet 8: e1002559, 2012.

18. Fransen K, Visschedijk MC, van Sommeren S, Fu JY, Franke L, Festen EA, Stokkers PC, van Bodegraven AA, Crusius JB Hommes DW, et al: Analysis of SNPs with an effect on gene expression identifies UBE2L3 and BCL3 as potential new risk genes for Crohn's disease. Hum Mol Genet 19: 3482-3488, 2010.

19. Montero-Melendez T, Llor X, Garcia-Planella E, Perretti M and Suárez A: Identification of novel predictor classifiers for inflammatory bowel disease by gene expression profiling. PLoS One 8: e76235, 2013

20. Vanhove W, Peeters PM, Staelens D, Schraenen A, Van der Goten J, Cleynen I, De Schepper S, Van Lommel L, Reynaert NL, Schuit F, et al: Strong upregulation of AIM2 and IFI16 inflammasomes in the mucosa of patients with active inflammatory bowel disease. Inflamm Bowel Dis 21: 2673-2682, 2015.

21. Parrish RS and Spencer HJ III: Effect of normalization on significance testing for oligonucleotide microarrays. J Biopharm Stat 14: 575-589, 2004

22. Langfelder $P$ and Horvath S: WGCNA: An R package for weighted correlation network analysis. BMC Bioinformatics 9: 559, 2008

23. Wang X, Kang DD, Shen K, Song C, Lu S, Chang LC, Liao SG, Huo Z, Tang S, Ding Y, et al: An R package suite for microarray meta-analysis in quality control, differentially expressed gene analysis and pathway enrichment detection. Bioinformatics 28 2534-2536, 2012.

24. Oughtred R, Chatraryamontri A, Breitkreutz BJ, Chang CS, Rust JM, Theesfeld CL, Heinicke S, Breitkreutz A, Chen D, Hirschman J, et al: BioGRID: A resource for studying biological interactions in yeast. Cold Spring Harb Protoc 2016: pdb. top080754, 2016.

25. Liu B and Hu B: HPRD: A high performance RDF database. Int J Parallel Emergent Distributed Systems 25: 123-133, 2010.

26. Szklarczyk D, Franceschini A, Wyder S, Forslund K, Heller D, Huerta-Cepas J, Simonovic M, Roth A, Santos A, Tsafou KP, et al: STRING v10: Protein-protein interaction networks, integrated over the tree of life. Nucleic Acids Res 43 (Database Issue): D447-D452, 2015

27. Kohl M, Wiese S and Warscheid B: Cytoscape: Software for visualization and analysis of biological networks. Methods Mol Biol 696: 291-303, 2011.

28. Dennis G Jr, Sherman BT, Hosack DA, Yang J, Gao W, Lane HC and Lempicki RA: DAVID: Database for annotation, visualization, and integrated discovery. Genome Biol 4: P3, 2003.

29. Gene Ontology Consortium: Gene ontology consortium: Going forward. Nucleic Acids Res 43 (Database Issue): D1049-D1056, 2015.

30. Kanehisa M, Sato Y, Kawashima M, Furumichi M and Tanabe $M$ KEGG as a reference resource for gene and protein annotation. Nucleic Acids Res 44: D457-D462, 2015.

31. Strickland DK, Au DT, Cunfer P and Muratoglu SC: Low-density lipoprotein receptor-related protein-1: Role in the regulation of vascular integrity. Arterioscler Thromb Vasc Biol 34: 487-498, 2014.

32. Fantus D, Awan Z, Seidah NG and Genest J: Aortic calcification: Novel insights from familial hypercholesterolemia and potential role for the low-density lipoprotein receptor. Atherosclerosis 226 $9-15,2013$

33. Ruan XZ, Varghese Z, Powis SH and Moorhead JF: Dysregulation of LDL receptor under the influence of inflammatory cytokines: A new pathway for foam cell formation. Kidney Int 60 : 1716-1725, 2001

34. Ren X, Shah TA, Ustiyan V, Zhang Y, Shinn J, Chen G, Whitsett JA, Kalin TV and Kalinichenko VV: FOXM1 promotes allergen-induced goblet cell metaplasia and pulmonary inflammation. Mol Cell Biol 33: 371-386, 2013.

35. Kim IM, Zhou Y, Ramakrishna S, Hughes DE, Solway J, Costa RH and Kalinichenko VV: Functional characterization of evolutionarily conserved DNA regions in forkhead box $\mathrm{f} 1$ gene locus. J Biol Chem 280: 37908-37916, 2005.

36. Feng Y, Wang L, Zeng J, Shen L, Liang X, Yu H, Liu S, Liu Z Sun Y, Li W, et al: FoxM1 is Overexpressed in Helicobacter pylori-induced gastric carcinogenesis and is negatively regulated by miR-370. Mol Cancer Res 11: 834-844, 2013.
37. Ziouzenkova O, Perrey S, Asatryan L, Hwang J, MacNaul KL Moller DE, Rader DJ, Sevanian A, Zechner R, Hoefler G and Plutzky J: Lipolysis of triglyceride-rich lipoproteins generates PPAR ligands: Evidence for an antiinflammatory role for lipoprotein lipase. Proc Natl Acad Sci USA 100: 2730-2735, 2003.

38. Tyagi S, Gupta P, Saini AS, Kaushal C and Sharma S: The peroxisome proliferator-activated receptor: A family of nuclear receptors role in various diseases. J Adv Pharm Technol Res 2: 236-240, 2011

39. Ooi EM, Watts GF, Sprecher DL, Chan DC and Barrett PH: Mechanism of action of a peroxisome proliferator-activated receptor (PPAR)-delta agonist on lipoprotein metabolism in dyslipidemic subjects with central obesity. J Clin Endocrinol Metab 96: E1568-E1576, 2011.

40. Herro R and Croft M: The control of tissue fibrosis by the inflammatory molecule LIGHT (TNF Superfamily member 14). Pharmacol Res 104: 151-155, 2016.

41. Liu TC, Naito T, Liu Z, VanDussen KL, Haritunians T, Li D, Endo K, Kawai Y, Nagasaki M, Kinouchi Y, et al: LRRK2 but not ATG16L1 is associated with Paneth cell defect in Japanese Crohn's disease patients. JCI Insight 2: e91917, 2017.

42. Serena C, Keiran N, Madeira A, Maymó-Masip E, Ejarque M, Terrón-Puig M, Espin E, Martí M, Borruel N, Guarner F, et al: Crohn's disease disturbs the immune properties of human Adipose-derived stem cells related to inflammasome activation. Stem Cell Reports 9: 1109-1123, 2017.

43. Kawai T and Akira S: Toll-like receptors and their crosstalk with other innate receptors in infection and immunity. Immunity 34 : 637-650, 2011.

44. Yuk JM and Jo EK: Toll-like receptors and innate immunity. J Bacteriol Virol 41: 225-235, 2011.

45. Frolova L, Drastich P, Rossmann P, Klimesova K and Tlaskalova-Hogenova H: Expression of Toll-like receptor 2 (TLR2), TLR4, and CD14 in biopsy samples of patients with inflammatory bowel diseases: Upregulated expression of TLR2 in terminal ileum of patients with ulcerative colitis. J Histochem Cytochem 56: 267-274, 2008

46. Netea MG, Kullberg BJ, de Jong DJ, Franke B, Sprong T, Naber TH, Drenth JP and Van der Meer JW: NOD2 mediates anti-inflammatory signals induced by TLR 2 ligands: Implications for Crohn's disease. Eur J Immunol 34: 2052-2059, 2004.

47. Szebeni B, Mraz M, Veres G, Dezsofi A, Vannay A, Vasarhelyi B, Majorova E, Arato A: Increased expression of toll-like receptor (Tlr) 2 and Tlr4 in the colonic mucosa of children with active inflammatory bowel disease. J Pediat Gastroenterol Nutrition 42: 34-41, 2006

48. Hausmann M, Kiessling S, Mestermann S, Webb G, Spöttl T, Andus T, Schölmerich J, Herfarth H, Ray K, Falk W and Rogler G: Toll-like receptors 2 and 4 are up-regulated during intestinal inflammation. Gastroenterology 122: 1987-2000, 2002.

49. Cosin-Roger J, Spalinger MR, Ruiz PA, Stanzel C, Terhalle A, Wolfram L, Melhem H, Atrott K, Lang S, Frey-Wagner I, et al: Gp96 deficiency affects TLR4 functionality and impairs ERK and p38 phosphorylation. PLoS One 13: e0193003, 2018.

50. Chandrasekharan B, Bala V, Kolachala VL, Vijay-Kumar M, Jones D, Gewirtz AT, Sitaraman SV and Srinivasan S: Targeted deletion of neuropeptide Y (NPY) modulates experimental colitis. PLoS One 3: e3304, 2008.

51. Hassani H, Lucas G, Rozell B and Ernfors P: Attenuation of acute experimental colitis by preventing NPY Y1 receptor signaling. Am J Physiol Gastrointest Liver Physiol 288: G550-G556, 2005.

52. Chandrasekharan B, Nezami BG and Srinivasan S: Emerging neuropeptide targets in inflammation: NPY and VIP. Am J Physiol Gastrointest Liver Physiol 304: G949-G957, 2013.

53. Vona-Davis LC and McFadden DW: NPY family of hormones: Clinical relevance and potential use in gastrointestinal disease. Curr Top Med Chem 7: 1710-1720, 2007.

54. El-Salhy M and Hausken T: The role of the neuropeptide Y (NPY) family in the pathophysiology of inflammatory bowel disease (IBD). Neuropeptides 55: 137-144, 2016.

This work is licensed under a Creative Commons Attribution-NonCommercial-NoDerivatives 4.0 International (CC BY-NC-ND 4.0) License. 